POS PROCEEDINGS

\title{
The TUS space experiment: calibration in flight
}

\author{
Alexandr Volvach ${ }^{1}$ \\ Crimean Astrophysical Observatory RAS, Yalta 98688 Crimea \\ E-mail: volvach@meta.ua
}

\author{
Alexandr Volvach, Andrei Dmitrotsa, Dmitrij Neyachenko \\ Crimean Astrophysical Observatory RAS, Yalta, Simeiz 298688 Crimea \\ V. Grebenyuk, N. Gorbunov, M. Lavrova, L. Tkachev \\ Joint Institute for Nuclear Research, Joliot-Curie, 6, Dubna, Moscow region, 141980 Russia,
}

\section{E. Popescu, Ana Caramete, Gabriel Chiritoi, Aurelian Andrei Radu}

Institute of Space Science, Magurele, Romania.

on behalf the Lomonosov-UHECR/TLE collaboration

\begin{abstract}
The TUS space experiment is aimed to study the energy spectrum and arrival direction of Ultra High Energy Cosmic Rays (UHECR) at E $\sim 10^{20} \mathrm{eV}$ by measuring the fluorescence yield of EAS in the atmosphere. The "Lomonosov" satellite, with TUS, was launched at the April 16, 2016. Satellite now is in orbit and fully operational. The TUS collaboration plans to design and produce a ground-based system of light sources for the in-flight calibration of the TUS detector. Two types of the possible UV light sources are presently under consideration for this purpose: LEDs and laser stations. The design and fabrication of the TUS ground-based calibration light source prototypes based on LedEngin-LZ4-00U600 LEDs and on the use of the "Simeiz-1873" laser stations are presented. Other calibration options are being studied: the use of signals from cosmicray interactions in the UV filter ahead of the photomultiplier, as well as other terrestrial sources of a different nature.
\end{abstract}




\section{Introduction}

The TUS detector was launched to the orbit with altitude $500 \mathrm{~km}$ on April 28, 2016. The TUS project task is experimental study of UHECR. The fluorescent and Cherenkov radiation of Extensive Air Showers (EAS) generated by UHECR particles will be detected at the night side of the Earth atmosphere from the orbital space platform at altitudes $400-500 \mathrm{~km}$. This will be possible to measure the $\mathrm{CR}$ spectrum, composition and arrival directions at $\mathrm{E}>7 \times 10^{19} \mathrm{eV}$ [1], [2] beyond the GZK energy limit. The SINP MSU, JINR and Space Regatta Consortium together with several Korean and Mexican Universities collaborate in the TUS detector preparation.

Important advantage of this detector will be a possibility to obtain data from the different directions of the sky with the same apparatus and with the same systematics. Besides the atmospheric conditions is more stable at the EAS research at space orbit as compared with ground based study. With such a data the difference between the results of the Auger detector in the south hemisphere and TA detector in north one may be understood. However the EAS signal at the space orbit is in $\sim 100$ times weaker that leads to a difficult problem due to background albedo radiation of the Earth atmosphere.

\section{The TUS detector.}

The EAS UV radiation is collected by the optical system of the TUS detector (Fig.1). There are two main parts of this: a modular Fresnel mirror and a photo receiver matrix with the corresponding DAQ electronics [3] (field of view (FoV) $\pm 4.5^{\circ}$, number of pixels 16x16 and light guides). One pixel is a Hamamatsu-type R1463 PMT with a 13-mm-diameter tube and UV glass window. A PMT quantum efficiency is $\sim 20 \%$ for the wavelength $350 \mathrm{~nm}$. The multi-alkali cathode was chosen for operation in linear regime in a wider range of temperatures. The Fresnel mirror has an area of $2.0 \mathrm{~m}^{2}$ and a focal distance of $1.5 \mathrm{~m}$. The DAQ electronics forms 256 channels with a time step $\Delta \mathrm{t}=0.8 \mu \mathrm{s}$. One pixel FoV is $\approx 0.1 \mathrm{msr}$, which corresponds to a spatial spot of $5 \times 5$ $\mathrm{km}^{2}$ on the Earth surface for the $500 \mathrm{~km}$ orbit height and $80 \mathrm{x} 80 \mathrm{~km}^{2}$ for the whole detector.

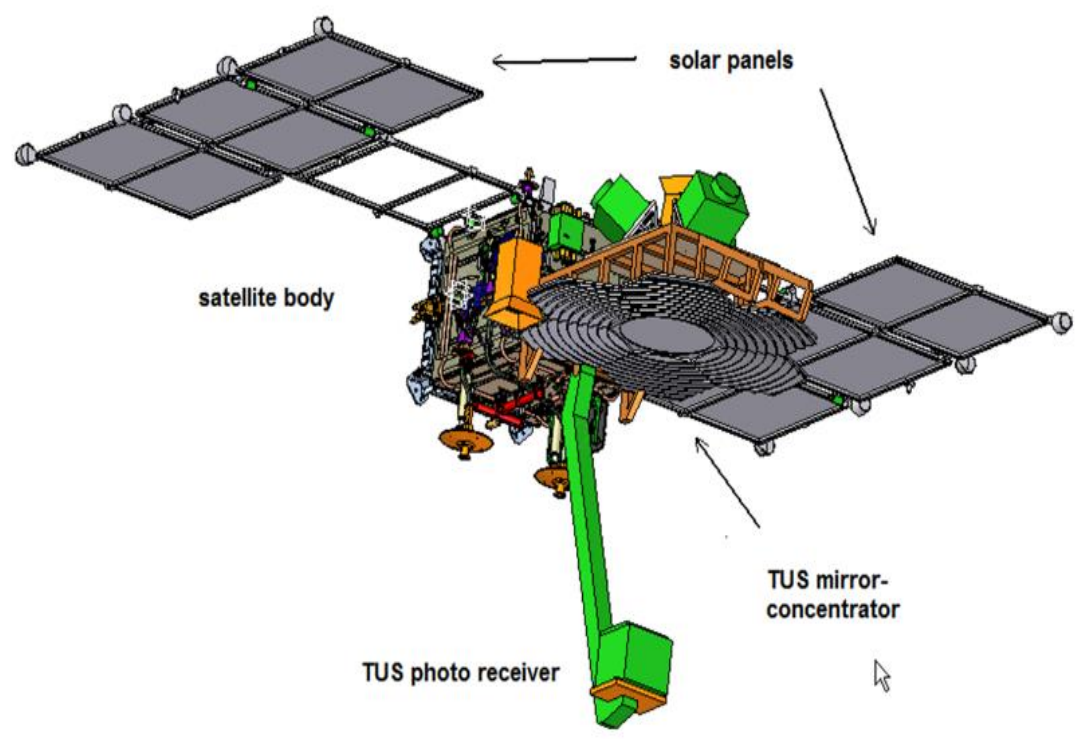

Figure 1. The TUS detector on board the Lomonosov satellite. 
The first months of the TUS operation in orbit were dedicated to the apparatus functionality measurements. During regular operation, the detector measures the UV background level and adjusts the HV and sensitivity of the PMTs to avoid their saturation under conditions of changed intensity of background radiation.

Table 1. Technical parameters of TUS.

\begin{tabular}{|c|c|}
\hline Parameter & Value \\
\hline Mass & $<60 \mathrm{~kg}$ \\
\hline Power (maximum) & $65 \mathrm{~W}$ \\
\hline Data (maximum) & 200 Mbyte/day \\
\hline FOV & $\pm 4,5$ degree \\
\hline Number of pixels & $256(16$ clusters of 16 PMTs $)$ \\
\hline Pixel size & $10 \mathrm{mrad}(5.5 \times 5.5 \mathrm{~km})$ \\
\hline Mirror area & $2.0 \mathrm{~m}^{2}$ \\
\hline Focal distance & $1.5 \mathrm{~m}$ \\
\hline Duty cycle & $30 \%$ \\
\hline
\end{tabular}

After flight tests and a tuning of TUS hardware and software on the satellite, the regular data are received since end of August 2016. Due to solar-synchronized orbit one week before and after of moonless time there is no Moon in the sky during the night part of the TUS orbit. It means that one got $~ 7000$ triggers/month. After removing the not moonless files the remaining data were analyzed through the Event Display program.

\section{Calibration technique}

The current TUS detector and electronics parameters may be measured and corrected online with such a calibration. The experimental technique and the measurement procedure are developing presently for this purpose. Two different technique is supposed to develop for the TUS photo detector calibration. The first one is to form the powerful LED or laser light beam with the pulse light intensity $>10^{15}$ photons $/ \mu$ s and the $300-400 \mathrm{~nm}$ spectral interval. The light beam is directed at some angle to the horizon and simulate EAS due to Reyleith scattering photons in air. Reyleith photons are propagate isotropically around of the beam axis and may be measered at the $\sim 500 \mathrm{~km}$ altitude with the TUS detector for calibration PMTs and trigger electronics. There is $10 \mathrm{sec}$ time interval for the optical contact between TUS and the calibration source at $\pm 4.5^{\circ}$ TUS angular FOV. Essential part of the PMT pixels may be irradiated and calibrated in sush case.

\subsection{Calibration with the powerful LED or laser light beam.}

Privilege of this approach is a possibility to illuminate and calibrate more PMT pixels due to extended light source during of one calibration run. As it was mention above it needs 100-500 photons $/\left(\mathrm{m}^{2} \mu \mathrm{s}\right)$ at the orbit altitude for calibration TUS photo detector. Therefore calibration with Reyleith scattering photons demands much more powerful light beam. Let's consider quantitatively the awaiting light flux at the space orbit. 
Track-like event registered on October 25, 2016 are presented in Fig.2.

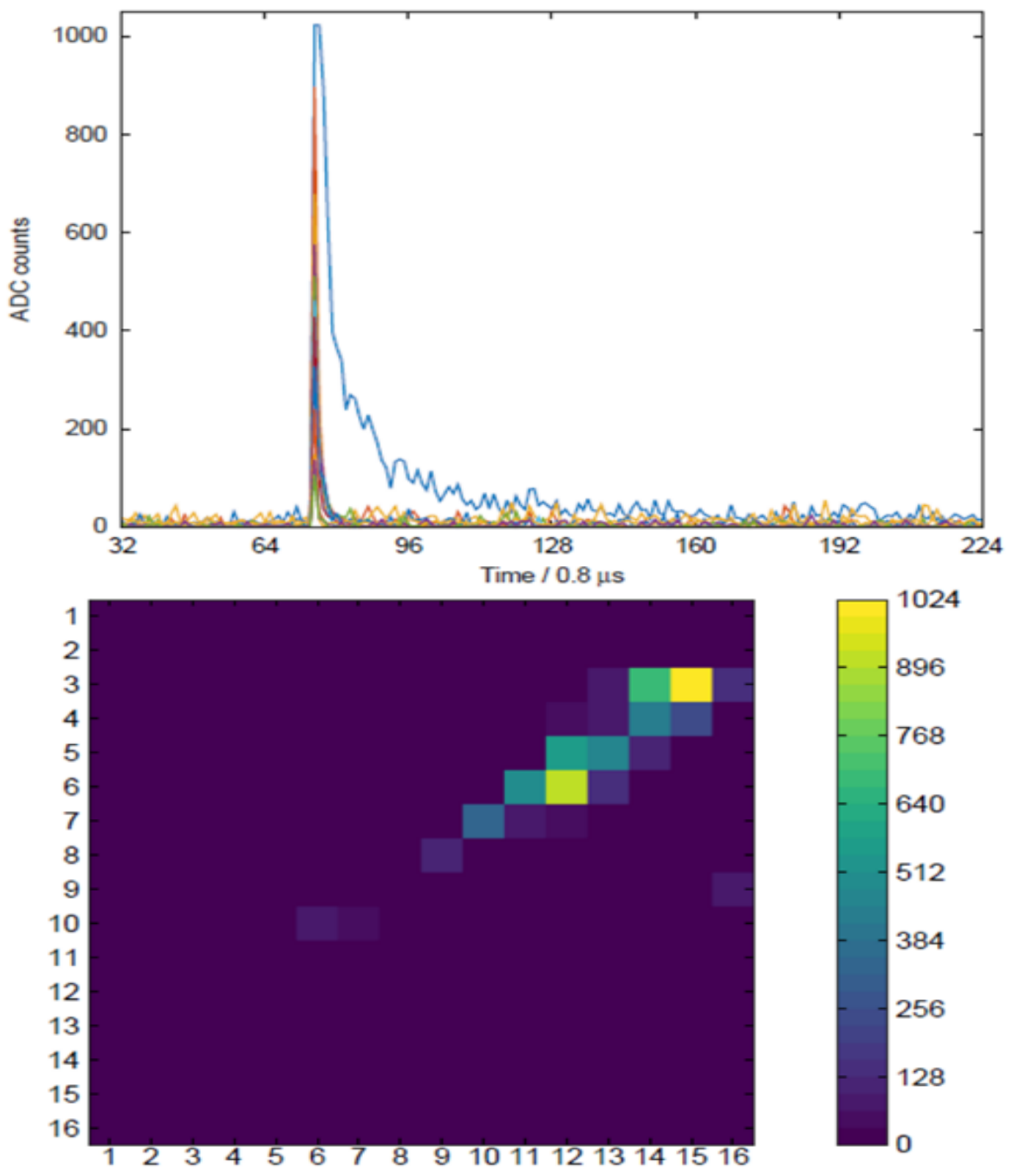

Figure 2. Track-like event registered on October 25, 2016. Top: Waveforms of ten PMTs that demonstrated the biggest ADC counts. Colors denote different pixels. Bottom: snapshot of the focal plane at the moment of maximum ADC counts.

At the first stage of the transmitter transformation, it is necessary to make changes to the optical scheme of the laser emitter (Fig.3):

1). To provide the necessary UV-range, it is required to introduce a third-harmonic generator (THG) into the radiator circuit, it can be obtained by combining two SHG crystals (355 nm). 
2). The optics of the range finder telescope should be adapted to the UV range. For the compression of the pulse, the SBS is included in the circuit; it is a compressor, MF, and two amplification cascades on YAG Nd + 3, and SHG provides radiation at a wavelength of $532 \mathrm{~nm}$.

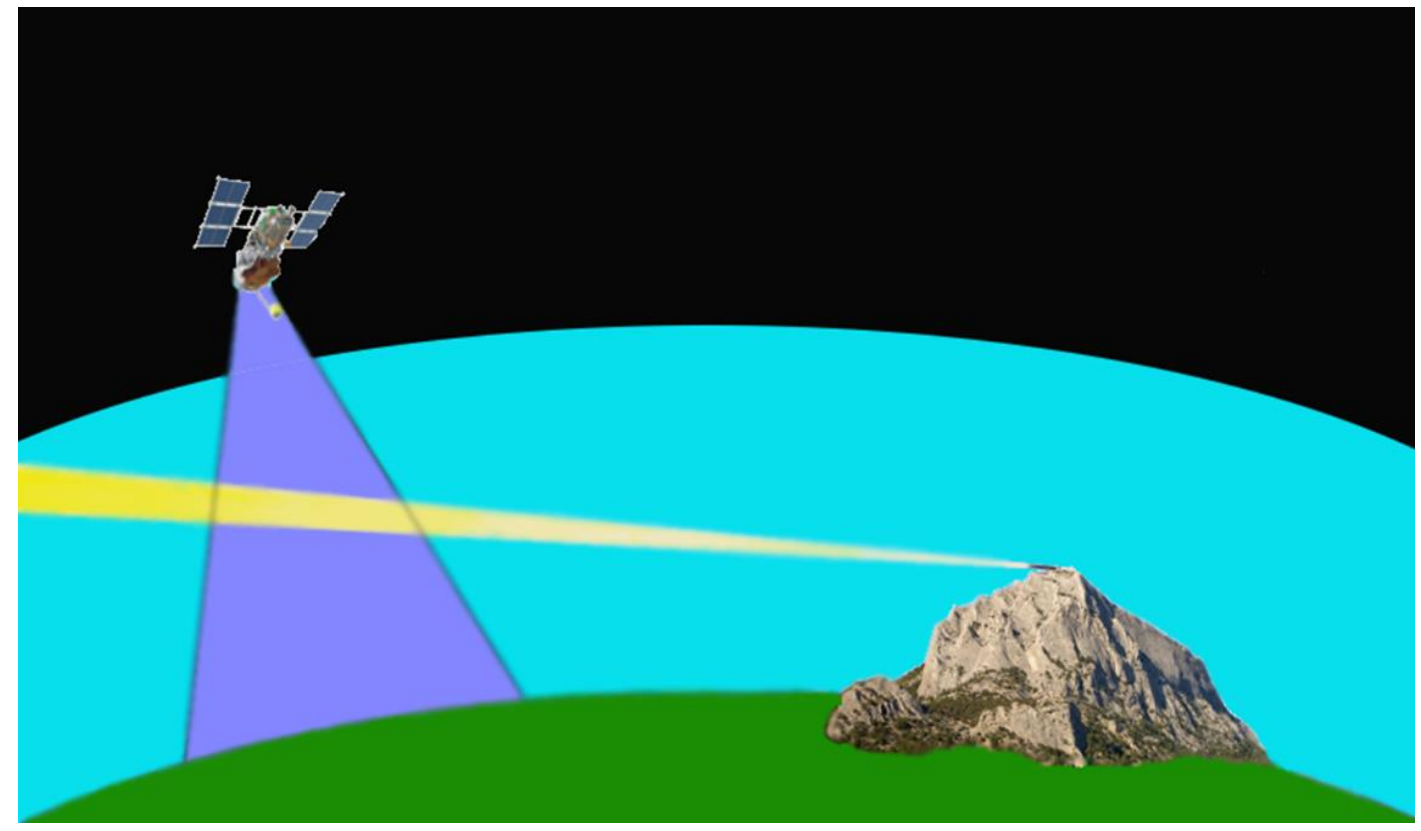

Figure 3. Schematic image of the photodetector calibration by an inclined beam.

The design and fabrication of the TUS ground-based calibration light source prototypes based on LedEngin-LZ4-00U600 LEDs and on the use of the "Simeiz-1873" laser stations are presented (Fig.4).
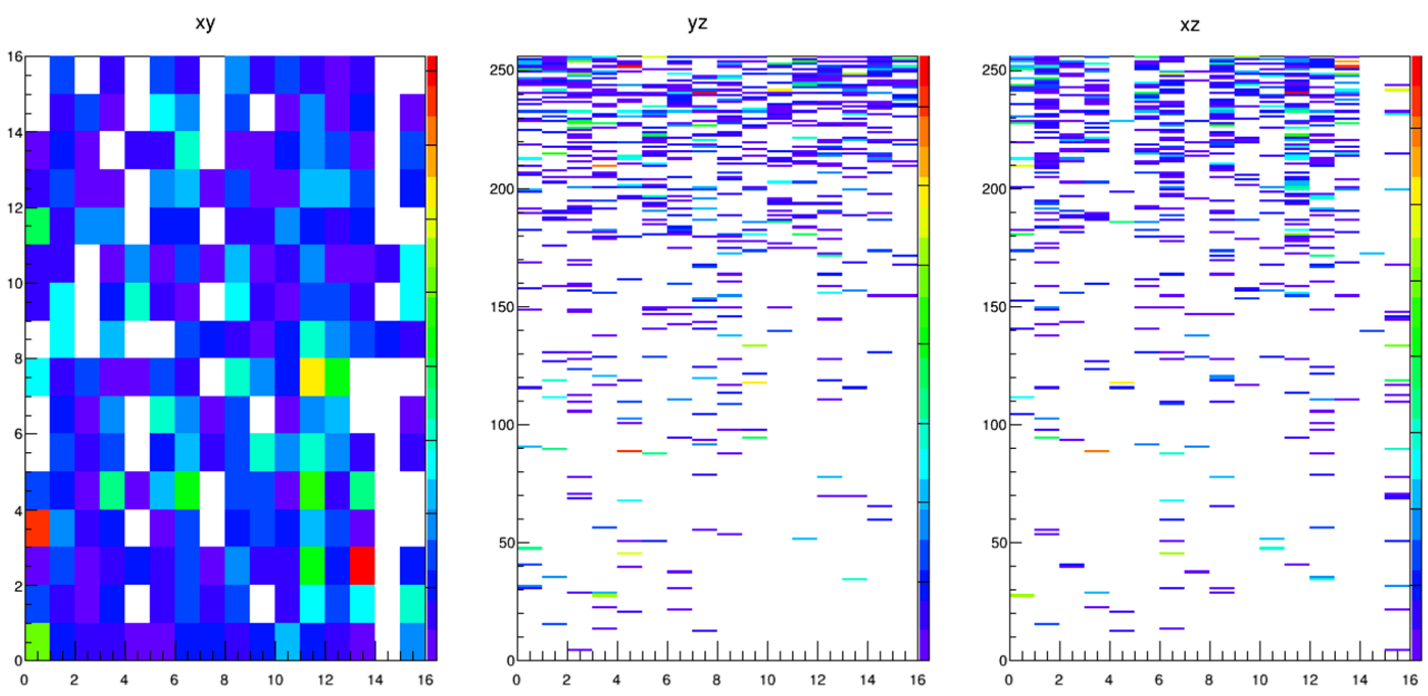

Figure 4. "Portrait" of the event, measured August 29, 2016 during the passage of the satellite over the Crimea. 
Other calibration options are being studied: the use of signals from cosmic-ray interactions in the UV filter ahead of the photomultiplier, as well as other terrestrial sources of a different nature.

\subsection{Calibration with the point LED beam}

In the second technique the LED beam is directed vertically and can be measured by the TUS photo detector if the calibration source is inside of $\pm 4.5^{\circ}$ TUS angular FOV. Calibration source image on photo detecor matrix may cross up to 16 PMTs and calibrated them during the satellite flight above.

Calibration source parameters were evaluated based on the Monte-Carlo simulation of the TUS detector where the optical system and trigger electronics characteristics were taken in account [4] - [7]:

1. Light pulse duration $-\geq 40 \mu \mathrm{s}$

2. The pulse energy $-10-50 \mathrm{~mJ}\left(1.9 \times 10^{16}-9.6 \times 10^{17}\right.$ photons $)$

3. The spectral interval $-300-400 \mathrm{~nm}$

4. The pulse frequency $-0.5-10 \mathrm{~Hz}$

5. Angular divergence of light beam $\pm 4.5^{\circ}$

6. Intensity of light flux at the $500 \mathrm{~km}$ latitude $100-500$ photons $/\left(\mathrm{m}^{2} \mu \mathrm{s}\right)$

7. Duration time of the calibration session - $10-100 \mathrm{sec}$

8. Long term stability of light source $1-3 \%$.

The source has to provide $100-500$ photons $/\left(\mathrm{m}^{2} \mu \mathrm{s}\right)$ for the reliable calibration. In the both techniques the difficult and unclear presently problem is an adequate consideration of the fluctuations of the photon absorbtion on the way from the ground source and the detector in orbit.

\subsection{Prototype source for TUS calibration}

Design and fabrication a prototype sourse for the TUS photo reciever calibration is presently under way. One research to use powerful LZ4-00U600 LED as one can see of LED Engin, Inc. company [8]. It is a high e-cacy $365 \mathrm{~nm}$ wave length LED suitable for calibration, optical power $11 \mathrm{~W}$, small size $7.0 \mathrm{~mm} \times 7.0 \mathrm{~mm}$ and integrated glass lens. The spectral power distribution is well suitable for the TUS calibration purposes.

\section{Conclusion}

1. The TUS mission aboard the dedicated Lomonosov satellite is the first mission to use an orbital UV telescope for the study of EASs produced by UHECR. The multifunctional "Lomonosov" satellite was launched on April 28, 2016. The satellite now is in the $500 \mathrm{~km}$ solarsynchronized orbit, operates and takes data, including the TUS measurements of EAS.

2. The ground-based calibration method is based on the use of high-power LEDs and laser beams are currently under development.

During 3-5 years of operation in space the TUS exposure will be $12000-20000 \mathrm{~km}^{2}$ _year_sr, comparable with the exposure of the largest ground-based detectors. Prototypes of the ground sources are in preparation for TUS photo receiver calibration in orbit. . Two types of the possible UV light sources are presently under consideration for this purpose: LEDs and laser stations. 


\section{Acknowledgments}

The TUS experiment on board the "Lomonosov" satellite was realized within the Federal Space Program of Russia with funding by the Russian Space Agency. The data analysis is supported by RFBR grants No. 16-29-13065 and No. 15-02-05498.

\section{References}

[1] B. A. Khrenov et al., Nucl. Phys. B (Proc. Suppl.) 112 (2002) 115.

[2] B. A. Khrenov et al., Phys. Atom. Nucl. 67(11) (2004) 2058.

[3] Abrashkin et al., Advances in Space Research 37 (2006) 1867.

[4] L. Tkatchev et al., 30th ICRC Proc.Merida (2007). Vol.5, p.881, ID 0162.

[5] A. Tkachenko et al., 32d ICRC Proc. Beijing 11 (2011) ID 272.

[6] G. Garipov et al., Phys. Part. Nuclei Lett. 10(1) (2013) 49.

[7] A. Tkachenko et all., 33d ICRC Proc. Rio De Janeiro (2013) ID 0423.

[8] http://www.ledengin.com/les/products/LZ4/LZ4-00U600.pdf 


\section{The Lomonosov-UHECR/TLE collaboration}

S.V. Biktemerova ${ }^{\mathrm{b}}$, A.A. Botvinkoc ${ }^{\mathrm{c}}$, A. I. Dmitrotsa ${ }^{\mathrm{g}}$, V.E. Eremeev ${ }^{\mathrm{a}}$, G.K. Garipov ${ }^{\mathrm{a}}$, V.M. Grebenyuk ${ }^{\mathrm{b}, \mathrm{d}}$, A.A. Grinyuk ${ }^{\mathrm{b}}$, S. Jeong ${ }^{\mathrm{e}}$, N.N. Kalmykova, M.A. Kaznacheeva ${ }^{\mathrm{a}}$, B.A. Khrenov ${ }^{\mathrm{a}}$, M. Kim ${ }^{\mathrm{e}}$, P.A. Klimov ${ }^{\mathrm{a}}$, M.V. Lavrova ${ }^{\text {, }}$ J. Lee ${ }^{\mathrm{f}}$, O. Martinez ${ }^{\mathrm{f}}$, M.I. Panasyuk ${ }^{\mathrm{a}}$, I.H. Park ${ }^{\mathrm{e}}$, V.L. Petrov ${ }^{\mathrm{a}}$, E. Ponce ${ }^{\mathrm{f}}$, A.E. Puchkov ${ }^{\mathrm{c}}$, H. Salazar ${ }^{f}$, O.A. Saprykin ${ }^{c}$, A.N. Senkovskyc , S.A. Sharakin ${ }^{\mathrm{a}}$, A.V. Shirokov ${ }^{\mathrm{a}}$, A.V. Tkachenko ${ }^{\mathrm{b}}$, L.G. Tkachev b;d, A. E. Volvach ${ }^{g}$, I.V. Yashin ${ }^{\text {a }}$, M.Yu. Zotova

${ }^{a}$ M.V. Lomonosov Moscow State University, GSP-1, Leninskie Gory, Moscow,119991, Russia

b Joint Institute for Nuclear Research, Joliot-Curie, 6, Dubna, Moscow region,Russia, 141980

${ }^{\text {c }}$ Space Regatta Consortium, ul. Lenina, 4a, 141070 Korolev, Moscow region,Russia

${ }^{\mathrm{d}}$ Dubna State University, University str., 19, Bld.1, Dubna, Moscow region, Russia

e Department of Physics and ISTS, Sungkyunkwan University, Seobu-ro 2066,Suwon,440-746 Korea

${ }^{\mathrm{f}}$ Benemerita Universidad Autonoma de Puebla, 4 sur 104 Centro Historico C.P.72000, Puebla, Mexico ${ }^{\mathrm{g}}$ Crimea Astrophysical Observatory RAS, Simeiz, Russia 